\title{
Taxonomie
}

\section{Welcher Coli ist ein Coli? Sequenzierung des Typstamms von Escherichia coli}

\author{
JAN P. MEIER-KOLTHOFF \\ LEIBNIZ-INSTITUT DSMZ - DEUTSCHE SAMMLUNG VON MIKROORGANISMEN UND \\ ZELLKULTUREN GMBH, BRAUNSCHWEIG
}

DOI: $10.1007 / \mathrm{s} 12268-015-0600-0$

(C) Springer-Verlag 2015

Für Hunderte von Stämmen von Escherichia coli sind Genomsequenzdaten mittlerweile öffentlich verfügbar. Umso überraschender ist, dass das Genom des Typstamms $\mathrm{U} 5 / 41^{\mathrm{T}}$ von E. coli erst vor Kurzem sequenziert wurde [1]. Typen sind notwendig für die taxonomische Nomenklatur. Wird ein Taxon gespalten, behält die den Typus enthaltende Untergruppe den originalen Namen; werden zwei Taxa vereinigt, wird der zum älteren Typus gehörende Name beibehalten. Der gelegentlich zu hörende Einwand, Typen seien nicht unbedingt die typischsten Vertreter ihres Taxons, trifft also nicht zu. Ohne den Vergleich mit dem jeweiligen Typstamm kann nicht mit Sicherheit gesagt werden, ob ein Stamm zu einer Bakterienart wie z. B. E. coli zählt.

\section{Genomsequenzdaten in der mikrobiellen Taxonomie}

In der traditionellen Taxonomie von Bakterien und Archaeen werden molekulare, chemotaxonomische und physiologische Eigenschaften verglichen. Dazu zählen die 16SrRNA-Gensequenz, die DNA:DNA-Hybridisierung (DDH) und der G+C-Gehalt. Viele dieser nasschemischen Verfahren sind arbeitsaufwendig und nur von Spezialisten anzuwenden. Genomsequenzen von Typstämmen ermöglichen hingegen alternative, bioinformatische Verfahren, die schnell und präzise durchzuführen und zu reproduzieren sind, da sie ausschließlich auf digitalen Daten beruhen $[2,3]$.

\section{Digitale DNA:DNA-Hybridisierung}

Die bedeutendste Methode ist hier die DDH, die seit mehr als 30 Jahren mittels eines definierten Schwellenwertes von 70 Prozent Ähnlichkeit über die Zugehörigkeit von Stämmen zur selben Art entscheidet [2]. Heute gibt es dafür eine moderne Alternative: die digitale
DDH (dDDH). Sie erlaubt eine Bestimmung der Artzugehörigkeit einer Genomsequenz in wenigen Minuten und steht Wissenschaftlern in Form des Webservice Genome-to-Genome Distance Calculator (GGDC) kostenlos unter http://ggdc.dsmz.de zur Verfügung [2].

Mithilfe des GGDC wurde das 5.038.133 Basenpaare lange Genom des E. coli-Typstamms mit etwa 250 anderen angeblichen E. coli-Genomen verglichen, deren Artzugehörigkeit bestätigt wurde [1]. Mit 99,2 Prozent DDH am ähnlichsten zum Typstamm ist der Krankheitserregerstamm S88. Die Plasmide beider Stämme sind nahezu identisch und zeigen eine hohe Übereinstimmung mit einem Plasmid des Krankheitserregers Salmonella enterica - ein deutlicher Hinweis auf den Austausch extrachromosomaler Elemente zwischen den Arten. Der bekannte Laborstamm K-12 zeigt dagegen eine deutlich geringere Ähnlichkeit von nur 75,2 Prozent DDH. Aus den GGDC-Ergebnissen kann leicht ein Stammbaum berechnet werden, der diesen Unterschied widerspiegelt (Abb. 1). Der E. coli-Typstamm unterscheidet sich zudem

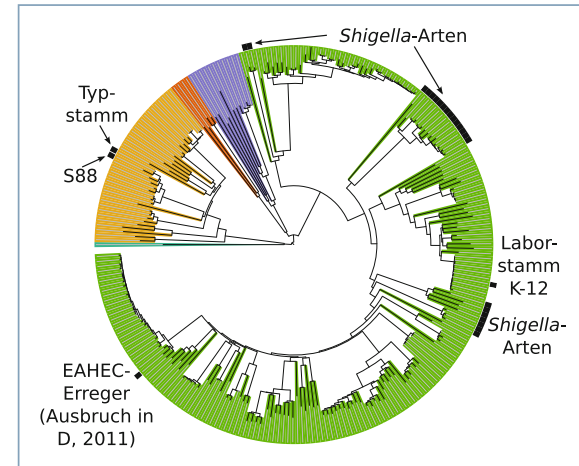

$\Delta$ Abb. 1: Vereinfachte Darstellung eines aus vollständigen Genomsequenzdaten von etwa 250 Escherichia coli-Stämmen erzeugten Stammbaums [1]. Die Einfärbung entspricht der neu vorgeschlagenen Einteilung in fünf Unterarten. Eine dieser Unterarten enthält sämtliche Stämme der Gattung Shigella, bekannt als Erreger der Shigellenruhr. auch physiologisch stark vom ungefährlichen Laborstamm K-12 [1].

\section{Traditionelle Fehler bei der Bestimmung des $\mathrm{G}+\mathrm{C}-$ Gehalts}

Für die Beschreibung von Arten und Gattungen wurde auch der $\mathrm{G}+\mathrm{C}-\mathrm{Gehalt}$ in der Vergangenheit mittels nasschemischer, indirekter Methoden bestimmt, woraus man folgerte, dass er innerhalb einer Art um bis zu fünf Prozent variieren kann. Seit der G+C-Gehalt mit viel höherer Genauigkeit direkt aus Genomsequenzen berechnet werden kann, weiß man seit Kurzem, dass er innerhalb von Arten um höchstens ein Prozent abweicht [3]. E. coli ist hier keine Ausnahme [1].

\section{Neue Methode zur Abgrenzung von Unterarten}

Bakterielle Unterarten beruhten traditionell auf ausgewählten phänotypischen Merkmalen. In der Arbeit über E. coli konnten wir einen Grenzwert von 79 Prozent dDDH-Ähnlichkeit vorschlagen, um auch Unterarten quantitativ vergleichbar zu machen [1]. Das Ergebnis ist in Abbildung $\mathbf{1}$ dargestellt und zeigt fünf Unterarten von E. coli. Dieses Verfahren lässt sich nun nutzen, um mittels des GGDC alle beliebigen bakteriellen Arten in Unterarten einzuteilen.

\section{Literatur}

[1] Meier-Kolthoff JP, Hahnke RL, Petersen J et al. (2014) Complete genome sequence of DSM $30083^{\mathrm{T}}$, the type strain $\left(\mathrm{U} 5 / 41^{\mathrm{T}}\right)$ of Escherichia coli, and a proposal for delineating subspecies in microbial taxonomy. Stand Genomic Sci 9:2 [2] Meier-Kolthoff JP, Auch AF, Klenk H-P et al. (2013) Genome sequence-based species delimitation with confidence intervals and improved distance functions.

BMC Bioinformatics 14:60

[3] Meier-Kolthoff JP, Klenk H-P, Göker M (2014) Taxonomic use of the $\mathrm{G}+\mathrm{C}$ content and DNA:DNA hybridization in the genomic age. Int J Syst Evol Microbiol 64:352-356

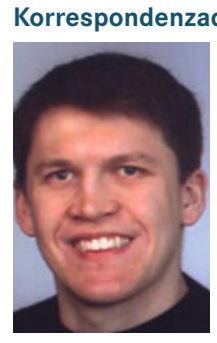

Dr. Jan P. Meier-Kolthoff AG Phylogenomik Leibniz-Institut DSMZ Deutsche Sammlung von Mikroorganismen und Zellkulturen $\mathrm{GmbH}$ Inhoffenstraße 7B D-38124 Braunschweig Tel.: 0531-2616-226 Fax: 0531-2616-418 jan.meier-kolthoff@dsmz.de 